\title{
Local States of Tellurium in Isolated Helical Chains
}

\author{
J. Stanek ${ }^{a}$, P. Fornal ${ }^{b}$, S.S. Hafner ${ }^{c}$ And A. Pfitzner ${ }^{d}$ \\ ${ }^{a}$ Institute of Physics, Jagiellonian University of Cracow \\ Reymonta 4, 30-059 Cracow, Poland \\ ${ }^{b}$ Institute of Physics, Technical University of Cracow, 30-084 Cracow, Poland \\ ${ }^{c}$ Center of Materials Sciences, University of Marburg, 35032 Marburg, Germany \\ ${ }^{d}$ Department of Inorganic Chemistry, University of Siegen, Germany
}

The local electronic state of $\mathrm{Te}$ in the helical chains of $\mathrm{CuXY}_{2(1-x)} \mathrm{Te}_{2 x}$ $(\mathrm{X}=\mathrm{Cl}, \mathrm{Br} ; \mathrm{Y}=\mathrm{Se}, \mathrm{S})$ were studied using the Mössbauer spectroscopy of ${ }^{125} \mathrm{Te}$ at $10 \mathrm{~K}$ and $70 \mathrm{~K}$. The isolated chains of $\mathrm{Te}$ in these compounds are less rigid than those in elemental Te. The determined data of quadrupole splitting $\Delta$ and Debye temperature $\Theta_{\mathrm{D}}$ are indicative of strong local ordering of $\mathrm{Te}$ in one of the two non-equivalent crystallographic positions. It was not possible to resolve crystallographic positions by the Mössbauer spectroscopy.

PACS numbers: $76.80 .+\mathrm{y}$

\section{Introduction}

Properties of elemental tellurium, Te, such as for example phase transformations from the hexagonal semiconducting phase to the monoclinic metallic phase at high pressures, have been interpreted in terms of the local Te bonding [1]. The crystal structure of elemental $\mathrm{Te}$ [2] consists of infinite helical chains which interact with each other. However, that strong interaction between the chains makes it rather difficult to estimate local bonding within the chains. In the present paper we should like to discuss some results obtained from samples of composition of $\mathrm{Cu}^{+} \mathrm{X}^{-} \mathrm{Y}_{2(1-x)} \mathrm{Te}_{2 x}$, where $\mathrm{X}=\mathrm{Cl}, \mathrm{Br} ; \mathrm{Y}=\mathrm{Se}, \mathrm{S}$ with the use of ${ }^{125} \mathrm{Te}$ Mössbauer spectroscopy. In these compounds the infinite TeY helical chains are, similar to elemental Te, the main structural elements. They are well separated in these cases, being held together by $\mathrm{Cu}^{+} \mathrm{X}^{-} \mathrm{Cu}^{+}$bridges. This may allow more direct insight into the distinct local electronic Te configuration which must lead to the formation of the chains. Further, an interesting aspect of $\mathrm{Cu}^{+} \mathrm{X}^{-} \mathrm{Y}_{2(1-x)} \mathrm{Te}_{2 x}$ compounds may 
be the question of possible atomic ordering within the helical chains over the Te, Y crystallographic positions, depending on the type of nearest neighbours within the chain or the type of connecting anion X. For such study, Mössbauer spectroscopy of ${ }^{125}$ Te may be an appropriate method. X-ray diffraction studies [3, 4] suggested strong ordering of Te and Y over two non-equivalent sites within the chain.

\section{Experimental}

\subsection{Samples}

The specimens for this study were synthesized by mixing $\mathrm{CuCl}, \mathrm{CuBr}, \mathrm{Te}$, $\mathrm{Se}, \mathrm{S}$ in appropriate proportions and heating in evacuated quartz capsules at temperatures of about $300^{\circ} \mathrm{C}$ for few weeks [4].

All compounds belong to the same structure type with the same structural topology. They crystallize in the monoclinic space group $P 2_{1} / n$. Projections of the crystal structure are shown in Figs. 1a and b. The chalcogen helical chains are parallel to the $b$-axis. They are held together by $\mathrm{Cu}-\mathrm{X}-\mathrm{Cu}$ units parallel to the $c$-axis. There are two non-equivalent crystallographic positions for Te and Y: $M 1$ and $M 2$, within each chain. These sites may be distinguished by their distances to the halide anions $\mathrm{X}$ or $\mathrm{Cu}$. For the $M 1$ position the distance $M 1-\mathrm{X}$ is shorter than for $M 2$, while for the $M 2$ position the $M 2-\mathrm{Cu}$ distance is shorter than for $M 1$. At first sight, $\mathrm{S}, \mathrm{Se}$, and Te may be expected to enter both sites, $M 1$ as well as $M 2$. However, a previous study [3, 4] suggested that the Te atoms preferentially occupy the $M 2$ position.

(a)
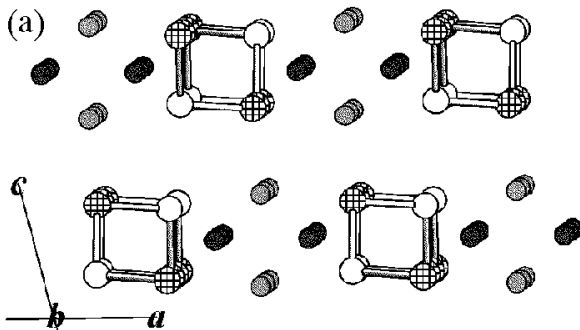

(b)

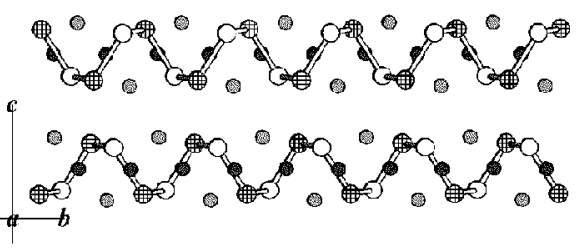

Fig. 1. (a) Projection of the crystal structure of $\mathrm{Cu}^{+} \mathrm{X}^{-} \mathrm{Y}_{2(1-x)} \mathrm{Te}_{2 x}(\mathrm{X}=\mathrm{Cl}, \mathrm{Br}$; $\mathrm{Y}=\mathrm{Se}, \mathrm{S})$ parallel to the helical chains of Te, Y. Solid circles: $\mathrm{Cu}$, shaded circles: $\mathrm{X}$, open circles: $M 1(\mathrm{Te}, \mathrm{Y})$, grating circles: $M 2$ (Te,Y) [4]. (b) Projection of the crystal structure of $\mathrm{Cu}^{+} \mathrm{X}^{-} \mathrm{Y}_{2(1-x)} \mathrm{Te}_{2 x}(\mathrm{X}=\mathrm{Cl}, \mathrm{Br} ; \mathrm{Y}=\mathrm{Se}, \mathrm{S})$ perpendicular to the helical chains of Te, Y. Atomic symbols as in part (a).

For further discussion three local configurations of Te atoms within one chain will be considered: configuration $\alpha$ refers to Te with two other Te as nearest neighbours: $-\mathrm{Te}-\mathbf{T e}-\mathrm{Te}-$, configuration $\beta$ refers to Te with two $\mathrm{Y}$ as nearest neighbours: $-\mathrm{Y}-\mathbf{T e}-\mathrm{Y}-$, and configuration $\gamma$ refers to Te with one Te and one $\mathrm{Y}$ as nearest 
neighbours: -Te-Te-Y-. It is to be anticipated that for our consideration of such configurations, it will be irrelevant whether the center position Te, or the neighboring Te, is located at $M 1$ or $M 2$ site.

\subsection{Mösbauer spectroscopy of ${ }^{125} \mathrm{Te}$}

Mössbauer spectroscopy of ${ }^{125} \mathrm{Te}$ is based on resonant absorption of $35.48 \mathrm{keV}$ $\gamma$ radiation which results from the decay of the first excited nuclear state, $I=+3 / 2$, to the ground state, $I=+1 / 2$. The relatively high energy of the transition requires usually measurement at low temperatures. The main disadvantage of ${ }^{125} \mathrm{Te}$ spectroscopy is the relatively broad natural line width $\Gamma=5.02 \mathrm{~mm} / \mathrm{s}$ at half maximum of the resonant absorption. Therefore, rather "poor resolution" is expected. Generally, $\Gamma$ is a few times larger than the range of observed isomer shifts, $\delta$. It is about 3 times smaller than the largest nuclear quadrupole splitting, $\Delta$, reported so far. A comprehensive review on ${ }^{125}$ Te Mössbauer spectroscopy is presented in [5]. In the following a few points relevant to the experiments reported here are described.

As source metastable ${ }^{125 \mathrm{~m}} \mathrm{Te}$ was used. $\mathrm{A} \mathrm{Mg}_{3}{ }^{124} \mathrm{TeO}_{6}$ matrix was activated at the Hahn-Meitner-Institut Berlin for 10 days in a neutron flux of $2 \times 10$ neutron $/\left(\mathrm{s} \cdot \mathrm{cm}^{2}\right)$. Due to the high Debye temperature, $\Theta_{\mathrm{D}}$, of $\mathrm{Mg}_{3} \mathrm{TeO}_{6}$ [6], it was possible to observe resonant absorption with source held at room temperature. The absorbing sample was mounted in a gas-flow cryostat. For the detection of the $35.48 \mathrm{keV}$ radiation, a germanium detector of highest purity was used. Alternatively, a proportional counter could also be employed, registering the "escape peak". The powdered absorbers were pressed to pellets which possessed a diameter of $1.0 \mathrm{~cm}$ and contained $20 \mathrm{mg}$ of natural tellurium per $\mathrm{cm}^{2}$ in each case. The effective absorber thickness, $t_{\mathrm{a}}$, calculated for one absorption line, was $t_{\mathrm{a}}=0.5$ at $10 \mathrm{~K}$ and $t_{\mathrm{a}}=0.2$ at $70 \mathrm{~K}$, obtained with the use of $\Theta_{\mathrm{D}}$ as determined (see section below). Thus, the approximation of thin absorber could be applied for the evaluation of the data.

\section{Results}

Spectra of all samples were recorded at $10 \mathrm{~K}$ and $70 \mathrm{~K}$. They exhibited the typical nuclear quadrupole-split doublet as shown in Fig. 2. The hyperfine parameters such as isomer shift $\delta$ referred to $\mathrm{Mg}_{3} \mathrm{TeO}_{6}$ at $295 \mathrm{~K}$, quadrupole splitting $\Delta$, line width $\Gamma$, and resonant absorption area $A$ are collected in Table.

The $\delta$ values were found to be the same for all the samples within the experimental error. For the $\mathrm{CuClSe}_{2(1-x)} \mathrm{Te}_{2 x}$ series $\Delta$ exhibited a significant decrease with increasing $x$ as shown in Fig. 3. For CuClSTe $\Delta$ is the same as for CuClSeTe while $\Delta$ for CuBrSTe is substantially smaller. It is interesting to note that the $\Delta$ values did not change between $10 \mathrm{~K}$ and $70 \mathrm{~K}$. However, $\Gamma$ showed at $10 \mathrm{~K}$ as well as at $70 \mathrm{~K}$ the same typical broadening with respect to its natural value. 


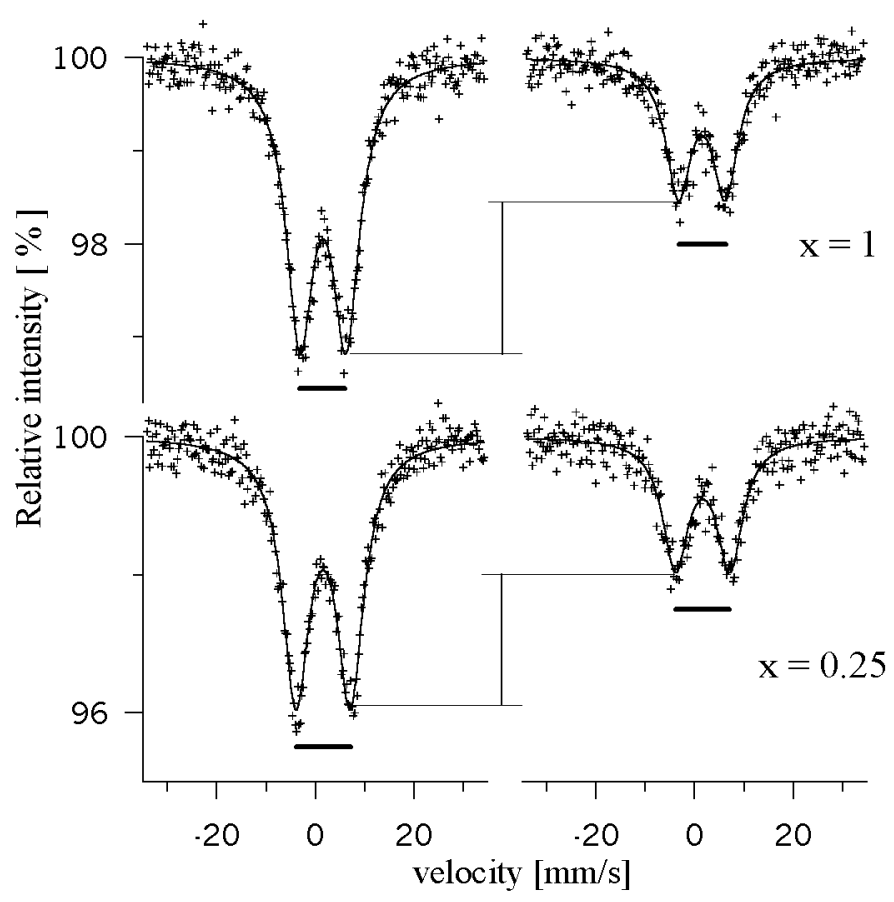

Fig. 2. Mössbauer spectra of ${ }^{125} \mathrm{Te}$ in $\mathrm{CuClSe}_{2(1-x)} \mathrm{Te}_{2 x}$. Upper spectrum $(x=1)$ at $10 \mathrm{~K}$ (left) and at $70 \mathrm{~K}$ (right), lower spectrum $(x=0.25)$ at $10 \mathrm{~K}$ (left) and at $70 \mathrm{~K}$ (right). Note the increased quadrupole splitting $\Delta$ with decreased fraction of Te. The decreasing intensity with increasing temperature is more pronounced for the sample richer in Te.

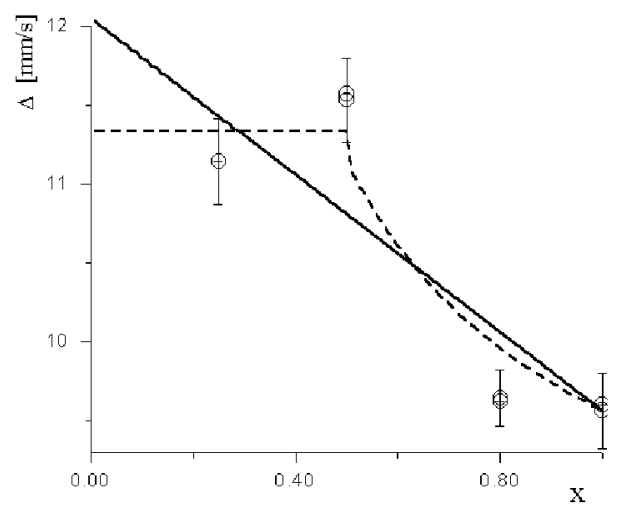

Fig. 3. Dependence of the quadrupole splitting $\Delta$ on $x$ in $\mathrm{CuClSe}_{2(1-x)} \mathrm{Te}_{2 x}$. Open circles refer to the experimental data of Table. The solid line is the dependence predicted assuming statistical distribution of Se, Te over $M 1$ and $M 2$. The dashed line refers to complete ordering of Te into $M 1$ or $M 2$. 
TABLE

${ }^{125}$ Te Mössbauer data of $\mathrm{Cu}^{+} \mathrm{X}^{-} \mathrm{Y}_{2(1-x)} \mathrm{Te}_{2 x}, \mathrm{X}=\mathrm{Cl}$, Br; Y $=\mathrm{S}$, Se. $T$ - temperature, $\delta$ - isomer shift referred to $\mathrm{Mg}_{3} \mathrm{TeO}_{6}$ source at $293 \mathrm{~K}$, $\Delta$ - nuclear quadrupole splitting, $\Gamma$ - full width at half maximum of the resonant absorption, $A-$ resonant absorption area, $\Theta_{\mathrm{D}}-$ Debye temperature.

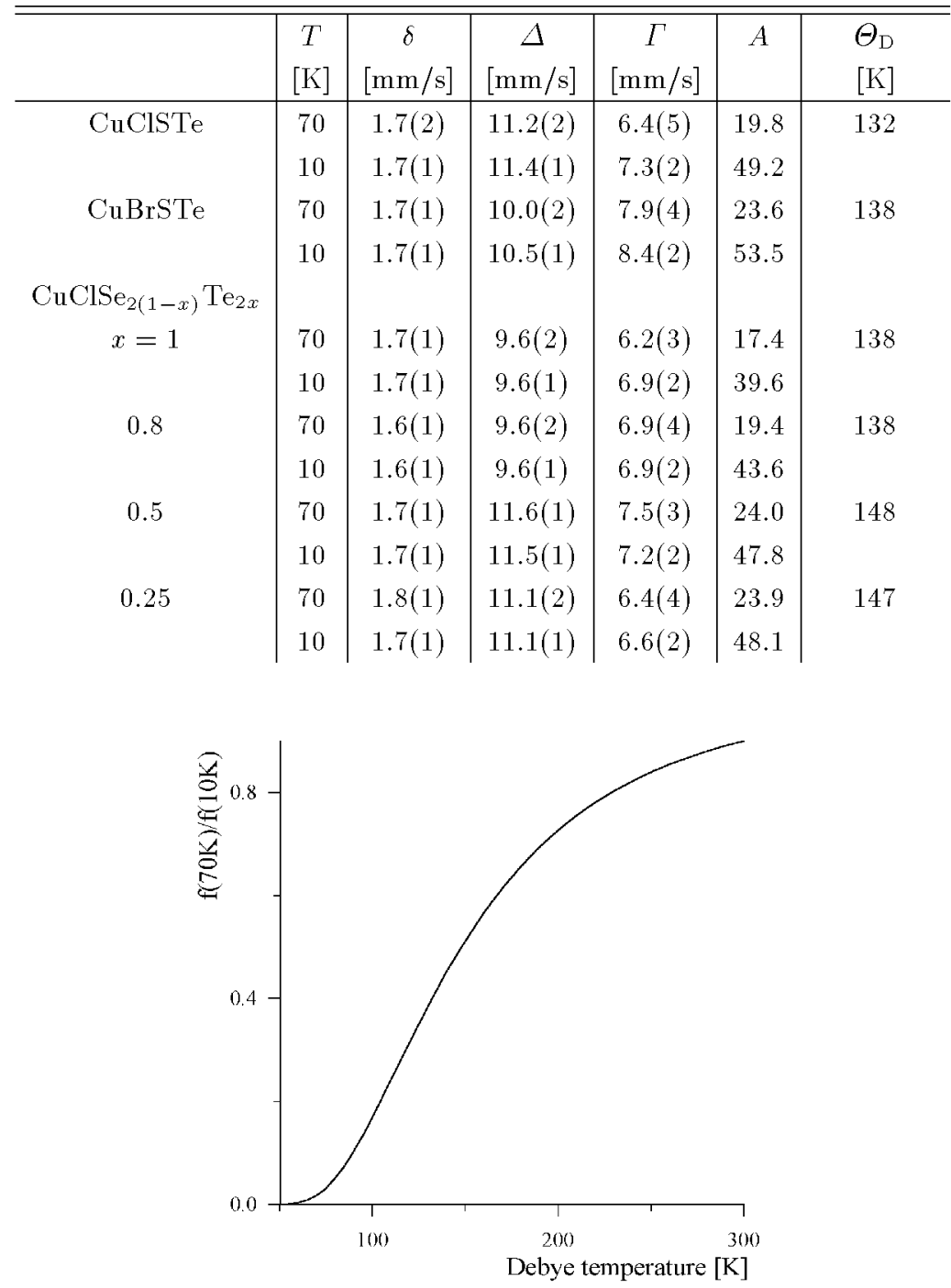

Fig. 4. Dependence of the ratio of ${ }^{125}$ Te recoil-free fraction $f_{70 \mathrm{~K}} / f_{10 \mathrm{~K}}$ on the Debye temperature $\Theta_{\mathrm{D}}$ used for determination of $\Theta_{\mathrm{D}}(x)$ values plotted in Fig. 5. 
The comparison of the resonant absorption area, $A$, of the spectra measured at two different temperatures in identical geometry allowed determination of the Debye temperature $\Theta_{\mathrm{D}}$ for each sample. The calculated ratio of $A(T=70 \mathrm{~K}) / A(T=10 \mathrm{~K})$ as function of $\Theta_{\mathrm{D}}$ for ${ }^{125} \mathrm{Te}$ is shown in Fig. 4. Using the experimental ratio $A(70 \mathrm{~K}) / A(10 \mathrm{~K}), \Theta_{\mathrm{D}}$ was obtained for each sample. The $\Theta_{\mathrm{D}}$ values are displayed in Table and Fig. 5. In case of the CuClSe $\mathrm{C}_{2(1-x)} \mathrm{Te}_{2 x}$ series an abrupt decrease in $\Theta_{\mathrm{D}}$ between $x=0.5$ and $x=0.8$ was observed.

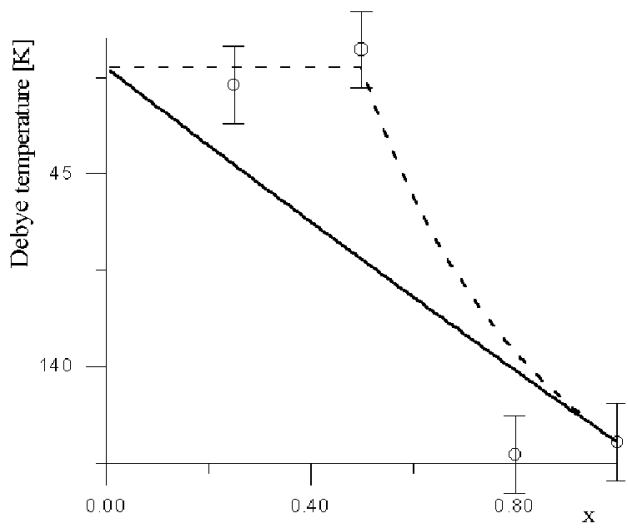

Fig. 5. Dependence of the Debye temperatures $\Theta_{\mathrm{D}}(x)$ on the Te fraction $x$ in $\mathrm{CuClSe}_{2(1-x)} \mathrm{Te}_{2 x}$. Open circles refer to the experimental data of Table. The solid line is the dependence predicted assuming statistical distribution of Se, Te over $M 1$ and $M 2$. The dashed line refers to complete ordering of Te into $M 1$ or $M 2$.

\section{Discussion}

The invariable value of $\delta$ in Table for all samples is not surprising. The difference in chemical bonding of $\mathrm{Te}$ in $\mathrm{Y}$ containing chains are, in principle, restricted to small differences in $5 p$ shell population, which may only indirectly influence the electron density at the Te nucleus. However, $\Delta$ is expected to be a quite sensitive measure of any anisotropy of $5 p$ shell.

\subsection{Comparison with elemental tellurium}

The bonding of $\mathrm{Te}$ in two-coordinated Te in Te bearing helical chains is predominantly determined by $5 p_{x}$ and $5 p_{y}$ orbital bonding parallel to the shortest M-M distances in the chain. However, the electric field gradient which determines the nuclear quadrupole splitting $\Delta$ results primarily from the lone pair of electrons in fully occupied Te $5 p_{z}$ orbitals parallel to the chain axis. It is interesting to note that $\Delta$ of elemental Te $(\Delta=7.68 \mathrm{~mm} / \mathrm{s}$ at $4.8 \mathrm{~K}, \Delta=7.36 \mathrm{~mm} / \mathrm{s}$ at $77 \mathrm{~K})$ 
is significantly smaller than $\Delta=9.6 \mathrm{~mm} / \mathrm{s}$ found in $\mathrm{CuClTe}_{2}$. This smaller $\Delta$ suggests some additional filling of the bonding $5 p_{x}, 5 p_{y}$ orbitals and, therefore, some additional bonding between Te chains. This conclusion is in agreement with our value $\Theta_{\mathrm{D}}=138 \mathrm{~K}$ for $\mathrm{CuClTe}_{2}$, which is remarkably lower than that for elemental Te $\left(\Theta_{\mathrm{D}}=180 \pm 15 \mathrm{~K}[7]\right)$. The decrease in $\Delta$ as reported for elemental Te between $4.8 \mathrm{~K}$ and $77 \mathrm{~K}$ has been interpreted as a result of torsional motions of the spiral chains. Such temperature dependence was not observed for our samples. Our results rather hint that torsion of isolated spiral chains held together by $\mathrm{Cu}^{+} \mathrm{Cl}^{-}$bridges is frozen.

\subsection{Ordering of Te in $\mathrm{CuClSe}_{2(1-x)} \mathrm{Te}_{2 x}$}

Our ${ }^{125} \mathrm{Te}$ spectra of $\mathrm{CuClTe}_{2}$ exhibited one doublet only, without notable line broadening. Distinct ${ }^{125}$ Te hyperfine splittings at the individual $M 1$ and $M 2$ sites could not be resolved. Therefore, it was not possible to identify $M 1$ and $M 2$ sites by ${ }^{125} \mathrm{Te}$ resonance. The geometrical distinction of $M 1$ and $M 2$ appears to be so small that any detection of intrinsic quadrupole splittings cannot be expected. The observed effective splittings $\Delta$ rather result from nearest $M 1$ and/or $M 2$ occupancies by Te or an Y atom other than Te, e.g. Se.

First, let us consider the hypothetical case of complete ordering for a particular chemical composition within the system $\mathrm{CuClSe}_{2(1-x)} \mathrm{Te}_{2 x}$. The probability $p(x)$ for the cluster $\alpha$ :-Te-Te-Te-.. within the chains is

$$
\begin{aligned}
& p_{\alpha}(x)=0 \quad \text { for } 0<x<0.5, \text { and } \\
& p_{\alpha}(x)=2 \cdot(x-0.5) \quad \text { for } 0.5<x<1
\end{aligned}
$$

for the cluster $\beta: \ldots-\mathrm{Se}-\mathbf{T e}-\mathrm{Se}-\ldots$, it is

$$
\begin{aligned}
& p_{\beta}(x)=1 \quad \text { for } 0<x<0.5, \text { and } \\
& p_{\beta}(x)=(2 / x) \cdot(1-x)^{2} \quad \text { for } 0.5<x<1,
\end{aligned}
$$

and finally for the cluster $\gamma: \ldots-\mathrm{Se}-\mathbf{T e}-\mathrm{Te}^{-} \ldots$, it is

$$
\begin{aligned}
& p_{\gamma}(x)=0 \quad \text { for } 0<x<0.5, \text { and } \\
& p_{\gamma}(x)=(2 / x) \cdot(1-x)(2 x-1) \quad \text { for } 0.5<x<1 .
\end{aligned}
$$

These probabilities are displayed in Fig. 6 (left). Accordingly, the respective probabilities $p$ for complete disordering, i.e. statistical distribution of Te over all $M 1$ and $M 2$ sites, are

$$
\begin{aligned}
& p_{\alpha}(x)=x^{2}, \\
& p_{\beta}(x)=(1-x)^{2}, \\
& p_{\gamma}(x)=2 x(1-x),
\end{aligned}
$$

as shown in Fig. 6 (right). 


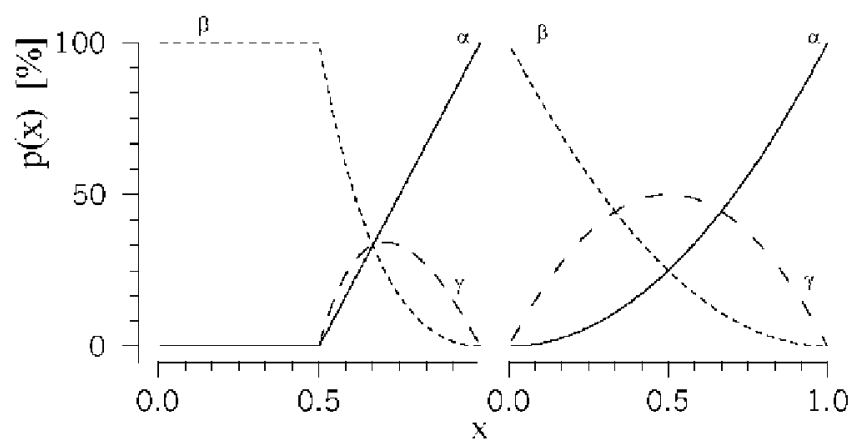

Fig. 6. Probabilities $p$ of the clusters $\alpha, \beta$, and $\gamma$ on tellurium concentration, $x$, for completely ordered phases (left) and completely disordered phases (right).

According to this simple model, the observed values $\Delta(x)$ in a binary system $\mathrm{CuXY}_{2(1-x)} \mathrm{Te}_{2 x}$ represent weighted averages of $\Delta_{\alpha}, \Delta_{\beta}$, and $\Delta_{\gamma}$, i.e. the individual quadrupole splittings for the clusters $\alpha, \beta$, and $\gamma$, respectively

$$
\Delta(x)=p_{\alpha}(x) \cdot \Delta_{\alpha}+p_{\beta}(x) \cdot \Delta_{\beta}+p_{\gamma}(x) \cdot \Delta_{\gamma} .
$$

The value of $\Delta_{\alpha}$ is known from the $\mathrm{CuClTe}_{2}$ spectrum: $\Delta_{\alpha}=9.6 \mathrm{~mm} / \mathrm{s}$ (Table). That of $\Delta_{\gamma}$ may be assumed generally as $\Delta_{\gamma}=0.5 \cdot\left(\Delta_{\alpha}+\Delta_{\beta}\right)$. From (10) $\Delta_{\beta}$ may be obtained using, in principle, just one value of $\Delta$ determined experimentally from a sample of arbitrary composition $x$ :

$$
\Delta_{\beta}[\mathrm{mm} / \mathrm{s}]=\left[2 \Delta-9.6\left(2 p_{\alpha}+p_{\gamma}\right)\right] /\left(2 p_{\beta}+p_{\gamma}\right) .
$$

In case of complete order, it is readily seen, within the approximation of this model, that Eq. (11) is reduced by insertion of Eqs. (1), (3), and (5) to $\Delta_{\beta}=\Delta(x)$ for any composition with $x<0.5$.

We may now compare the experimental quadrupole splittings $\Delta$ of the system $\mathrm{CuClSe}_{2(1-x)} \mathrm{Te}_{2 x}$ reported in Table with $\Delta$ derived from our simple model for completely ordered phases as well as those for completely disordered ones by insertion of the appropriate probability terms $p$ defined in Eqs. (1) to (9) into Eqs. (10) and (11). First, the completely ordered phases will be considered. Inserting the experimental $\Delta$ data for $x=0.25$ and $x=0.50$ into (11) and using the $p$ values of Eqs. (1), (3), and (5) results in the model values $\Delta_{0.25}=11.1 \mathrm{~mm} / \mathrm{s}$ and $\Delta_{0.5}=11.5 \mathrm{~mm} / \mathrm{s}$, respectively. Inserting the experimental $\Delta$ for $x=0.80$ into (11) and $p$ of Eqs. (2), (4), and (6) accordingly results in the model value $9.6 \mathrm{~mm} / \mathrm{s}$.

$\Delta(x)$ for the completely disordered phases may be calculated with the use of Eqs. (7), (8), (9), and (10):

$$
\begin{aligned}
& \Delta(x)[\mathrm{mm} / \mathrm{s}]=9.6 x^{2}+\Delta_{\beta}(1-x)^{2}+\left(9.6+\Delta_{\beta}\right) x(1-x), \text { or } \\
& \Delta_{\beta}=(\Delta-9.6 x) /(1-x) .
\end{aligned}
$$

Equation (13) is valid for the entire range of compositions $x(0<x<1)$. 
The same procedure was applied for the experimentally determined values $\Theta_{\mathrm{D}}(x)$. The predicted, resulting dependence of $\Theta_{\mathrm{D}}$ on the concentration $x$ is illustrated in Fig. 5. The principal similarity of Figs. 3 and 5 should be noted in view of the non-correlated determination of $\Delta$ and resonant absorption areas $A$ (Table). Comparison of measured and predicted $\Theta_{\mathrm{D}}(x)$ as well as the $\Delta(x)$ values, based on our simple model of local clustering, favours strongly local ordering of Te over one of the $\mathrm{M}$ sites, which may be $M 1$ or $M 2$. As mentioned at the beginning of this section, it is not possible to distinguish between $M 1$ and $M 2$ from ${ }^{125} \mathrm{Te}$ spectroscopy alone.

\subsection{The role of chalcogen and halide anions}

The substitution of Se by $\mathrm{S}$ does not change the electronic state of Te significantly as concluded from the ${ }^{125} \mathrm{Te}$ spectra of CuClSTe. However, further replacement of $\mathrm{Cl}$ by $\mathrm{Br}$ (Table) reduces the value of $\Delta$. This is indicative of some additional electron transfer from $\mathrm{Br}$ to the $5 p_{x}, 5 p_{y}$ orbitals of Te. The $\mathrm{Y}-\mathrm{X}$ distance appears to be shorter at the $M 2$ site than at the $M 1$ site. Thus, the influence of $\mathrm{Br}$ substitution for $\mathrm{Cl}$ on the electronic Te state, indeed, suggests that Te is ordered into the $M 2$ position as it was concluded from X-ray diffraction.

\section{Acknowledgment}

We thank Dorothea Alber, Hahn-Meitner-Institut Berlin for activating the ${ }^{125 \mathrm{~m}}$ Te sources. This work was partly financed by the project WTZ POL 98-031 of DLR (BMBF), Germany.

\section{References}

[1] P. Vulliet, J.P. Sanchez, unpublished.

[2] C.E. Violet, R. Booth, Phys. Rev. 144, 225 (1986).

[3] A. Pfitzner, S. Zimmerer, Z. Anorg. Allg. Chem. 621, 969 (1995).

[4] A. Pfitzner, S. Zimmerer, Z. Anorg. Allg. Chem. 622, 853 (1995).

[5] F.J. Berry, in: Mössbauer Spectroscopy Applied to Inorganic Chemistry, Vol. 2, Ed. G.J. Lang, Plenum Press, New York 1987, p. 343.

[6] H. Binczycka, S.S. Hafner, J. Stanek, M. Tromel, Phys. Lett. A 131, 135 (1988).

[7] P. Booland, B.L. Robinson, S. Jha, Phys. Rev. B 2, 3463 (1970). 